\title{
Aptamer-based single-molecule imaging of insulin receptors in living cells
}

Minhyeok Chang

Mijin Kwon

Sooran Kim

$\mathrm{Na}-\mathrm{Oh}$ Yunn

Daehyung Kim

Sung Ho Ryu

Jong-Bong Lee 


\title{
Aptamer-based single-molecule imaging of insulin receptors in living cells
}

\author{
Minhyeok Chang, ${ }^{a^{*}}$ Mijin Kwon, ${ }^{b^{*}}$ Sooran Kim, ${ }^{a^{*}}$ Na-Oh Yunn, ${ }^{c^{*}}$ Daehyung Kim, ${ }^{\text {a Sung Ho Ryu, }}$,e and Jong-Bong Lee ${ }^{\mathrm{a}, \mathrm{c}}$ \\ aPohang University of Science \& Technology, Department of Physics, Pohang 790-784, Republic of Korea \\ bUlsan National Institute of Science \& Technology, Department of Biological Science, Ulsan, Republic of Korea \\ 'Pohang University of Science \& Technology, School of Interdisciplinary Bioscience \& Bioengineering, Pohang 790-784, Republic of Korea \\ dPohang University of Science \& Technology, Department of Life Sciences, Pohang 790-784, Republic of Korea \\ ePohang University of Science \& Technology, Integrative Biosciences \& Biotechnology, Pohang 790-784, Republic of Korea
}

\begin{abstract}
We present a single-molecule imaging platform that quantitatively explores the spatiotemporal dynamics of individual insulin receptors in living cells. Modified DNA aptamers that specifically recognize insulin receptors (IRs) with a high affinity were selected through the SELEX process. Using quantum dot-labeled aptamers, we successfully imaged and analyzed the diffusive motions of individual IRs in the plasma membranes of a variety of cell lines (HIR, HEK293, HepG2). We further explored the cholesterol-dependent movement of IRs to address whether cholesterol depletion interferes with IRs and found that cholesterol depletion of the plasma membrane by methyl- $\beta$ cyclodextrin reduces the mobility of IRs. The aptamer-based single-molecule imaging of IRs will provide better understanding of insulin signal transduction through the dynamics study of IRs in the plasma membrane. $\odot$ The Authors. Published by SPIE under a Creative Commons Attribution 3.0 Unported License. Distribution or reproduction of this work in whole or in part requires full attribution of the original publication, including its DOI. [DOI: 10.1117/1.JBO.19.5.051204]
\end{abstract}

Keywords: aptamer; total internal reflection fluorescence microscopy; single-molecule imaging; insulin receptor; diffusion; cholesterol. Paper 130540SSPR received Jul. 30, 2013; revised manuscript received Oct. 7, 2013; accepted for publication Oct. 28, 2013; published online Dec. 2, 2013.

\section{Introduction}

A variety of molecular dynamics and interactions that are inherently heterogeneous is involved in cellular processes. Most cellular events are initiated by ligand-receptor interactions at the plasma membrane. The interactions and dynamics of these complexes may require only small populations of molecules and transient interactions that cannot be detected by conventional ensemble average measurements. To overcome such limitations, single-molecule fluorescence microscopy has been widely employed to quantitatively detect the spatiotemporal dynamics of individual proteins and their interactions in live cells with high sensitivity. ${ }^{1,2}$

To visualize individual membrane proteins, the proteins are coupled to antibodies conjugated to fluorescent probes, such as organic fluorophores or semiconductor nanocrystals, known as quantum dots (QDs). ${ }^{3,4}$ Another widely used approach is genetic fusion of a fluorescent protein and the protein of interest. ${ }^{5}$ However, fluorescent proteins show poor photostability, which limits the time trajectory length in single-particle tracking experiments. Aptamers are small oligonucleotides obtained through an in vitro selection process, which is optimized to bind a target protein with a high affinity, similar to that of antibodies. ${ }^{6,7}$ Aptamers can be substituted for antibodies due to their remarkable advantages including easy modification for labeling fluorophores and smaller size. ${ }^{8}$

\footnotetext{
These authors equally contributed.

Address all correspondence to: Jong-Bong Lee, Pohang University of Science \& Technology, Department of Physics, Pohang 790-784, Republic of Korea. Tel: 8254-279-2095; Fax: 82-54-279-3099; E-mail: jblee@postech.ac.kr; or Sung Ho Ryu, Pohang University of Science \& Technology, Department of Life Sciences, Pohang 790-784, Republic of Korea. Tel: 82-54-279-2292; Fax: 8254-279-0645; E-mail: sungho@postech.ac.kr
}

We used modified DNA aptamers to monitor the dynamics of insulin receptors (IRs) in living cells. IRs have been extensively studied for decades due to their important role in the regulation of glucose homeostasis. ${ }^{9}$ In particular, the cholesterol-dependent signaling of IRs has been suggested for a model of glucose uptake in metabolic cells. ${ }^{10}$ Despite the biological importance of IRs and numerous related studies including single-molecule imaging by electron microscopy ${ }^{11}$ and atomic force microscopy, ${ }^{12}$ the real-time dynamics of individual IR in living cells has not yet been reported. We monitored the diffusive motions of human IRs in living cells by imaging individual human IRs coupled to fluorescently labeled aptamers or insulin ligands using total internal reflection fluorescence (TIRF) microscopy. This single-molecule study allowed us to explore the mechanistic properties of IRs at the plasma membrane, including the cholesterol-dependent mobility of IRs.

\section{Materials and Methods}

\subsection{Cell Preparation}

HEK293T, HepG2, and human IR-overexpressing Rat-1 (HIR) cells were selected as appropriate cell lines for this study because their adhesive growth on surfaces is suitable for TIRF microscopy. HEK293 cell line, which is derived from human embryonic kidney cells, has been extensively used in cell biology research. HepG2 cell line was derived from human liver tissue and is involved in signal transduction including glucose homeostasis. HIR cells can adequately increase the specificity of our observations by providing enough receptors for the probes to bind. The selected cell lines were cultured for 24 to $48 \mathrm{~h}$ on a circular cover glass (Corning No. 1.5, Fisher Scientific; $r=25 \mathrm{~mm}$ ) in $1 \times$ Dulbecco's modified eagle medium (DMEM), high glucose (Invitrogen, Carlsbad, 
California) containing $10 \%$ fetal bovine serum (FBS), at $37^{\circ} \mathrm{C}$ and $5 \% \mathrm{CO}_{2}$; the cells were then observed for the experiments. Before the cells were seeded, the cover glass was stringently cleaned twice by sonication with ethanol and $\mathrm{KOH}$ for $30 \mathrm{~min}$, respectively, to reduce autofluorescence from the cover glass and nonspecific binding of the aptamers and insulin ligands to the cover glass. The cell-cultured cover glass was placed in a Chamlide magnetic chamber (Live Cell Instruments, Korea) to image IRs at the membrane.

\subsection{Measurement of Dissociation Constants}

Binding studies were performed as previously described. ${ }^{13}$ To determine the dissociation constants $\left(K_{d}\right)$, the amount of aptamer bound to the IR recombinant proteins (R\&D Systems, Minneapolis, Minnesota) was measured using $\left[\alpha-{ }^{32} \mathrm{P}\right]$-ATP-labeled aptamer $(3000 \mathrm{Ci} / \mathrm{mmol}, \quad 10 \mathrm{mCi} / \mathrm{ml}$; Perkin Elmer, Waltham, Massachusetts) in a selection buffer (40 mM HEPES, pH 7.2, $102 \mathrm{mM} \mathrm{NaCl,} 5 \mathrm{mM} \mathrm{MgCl}_{2}$, $5 \mathrm{mM} \mathrm{KCl})$ at $37^{\circ} \mathrm{C}$. The $K_{d}$ values of the aptamers were determined by measuring the fraction of aptamers bound to the proteins under various recombinant protein concentrations (10 pM to $100 \mathrm{nM})$.

\subsection{Western Blot Analysis}

HIR cells were serum starved in DMEM for $4 \mathrm{~h}$ at $37^{\circ} \mathrm{C}$. After the cells were stimulated with insulin or aptamers for $15 \mathrm{~min}$, the medium was immediately removed and the cells were washed out with ice-cold phosphate buffered saline (PBS) buffer. The cells were lysed in lysis buffer containing $50 \mathrm{mM}$ Tris- $\mathrm{HCl}$, pH 7.4, $150 \mathrm{mM} \mathrm{NaCl}, 1 \mathrm{mM}$ EDTA, $10 \mathrm{mM} \beta$-glycerophosphate, $50 \mathrm{mM} \mathrm{NaF}, 1.5 \mathrm{mM}$ sodium orthovanadate, and $1 \%$ Triton-X. Cell debris was removed by centrifugation for $15 \mathrm{~min}$ at $16,000 \times \mathrm{g}$ at $4^{\circ} \mathrm{C}$, and the protein concentration of the supernatants was measured using a BCA protein assay kit (Pierce, Rockford, Illinois). The cell lysates were boiled for $10 \mathrm{~min}$ at $95^{\circ} \mathrm{C}$ in the sample buffer and were then resolved by sodium dodecyl sulfate-polyacrylamide gel electrophoresis (SDS-PAGE). The gels were transferred to a nitrocellulose membrane, and the membrane was blocked in TTBS buffer containing $10 \mathrm{mM}$ Tris-Cl, pH 7.4, $150 \mathrm{mM} \mathrm{NaCl}, 0.05 \%$ Tween 20, and 5\% nonfat dry milk. After an overnight incubation with specific primary antibodies at $4{ }^{\circ} \mathrm{C}$ and incubation with IRDye800-conjugated anti-rabbit or anti-mouse IgG secondary antibodies for $1 \mathrm{~h}$ at room temperature, the blots were visualized by an Odessay infrared imaging system (LI-COR Bioscience, Lincoln, Nebraska). Anti-IR antibody, anti-phospho IR (pY1150/pY1151) antibody, and anti-phospho IRS1 (pY632) antibody were purchased from Santa Cruz Biotechnology (Dallas, Texas). Anti-phospho AKT (pS473) was purchased from Cell Signaling Technology (Danvers, Massachusetts).

\subsection{Flow Cytometry}

HIR cells were cultured in DMEM with $10 \%$ FBS and were suspended in $5 \mathrm{mM}$ EDTA-PBS solution. The suspended cells were collected by centrifugation for $2 \mathrm{~min}$ at $320 \times \mathrm{g}$ and were then resuspended using Hanks balanced salt solution (HBSS). A total of $2 \times 10^{4}$ cells $/ \mathrm{ml}$ was stained using $200 \mathrm{nM} \mathrm{Cy3-conjugated}$ aptamer with or without $1 \mu \mathrm{M}$ insulin for $1 \mathrm{~h}$ at $4^{\circ} \mathrm{C}$. After three washing steps, the cells were fixed in HBSS containing $1 \%$ paraformaldehyde. The cells were run on a FACSCalibur (BD Biosciences, San Jose, California).

\subsection{Sample Preparation for Single-Molecule Imaging}

Before the conjugated aptamers were injected into the sample chamber, the aptamers were heated at $85^{\circ} \mathrm{C}$ for $5 \mathrm{~min}$ and then slowly cooled to room temperature to activate their structure. The active aptamers formed by heating were diluted to $28 \mathrm{nM}$ with $98 \mu \mathrm{M}$ Dextran (Sigma-Aldrich, St. Louis, Missouri) in $1 \times$ DMEM and were incubated with the cells for $15 \mathrm{~min}$ at $37^{\circ} \mathrm{C}$ in the presence of $5 \% \mathrm{CO}_{2}$. Dextran, which is generally used to increase the specificity of aptamers in the SELEX process, ${ }^{6,7}$ was added to reduce the nonspecific binding of aptamers to the cover glass in this step. For the experiment using QD-labeled aptamers, 0.5 nM streptavidin-coated QDs (QD605, Invitrogen) in $1 \times$ DMEM with $0.3 \mathrm{mg} / \mathrm{ml}$ bovine serum albumin (BSA, Sigma-Aldrich) was added to the sample chamber, which had been incubated with DMEM-BSA solution for 10 to $30 \mathrm{~min}$ after aptamer binding. The free aptamers and QDs in solution were removed by pipetting three times before imaging to observe specific aptamers binding to IRs only and to reduce background noise. For control experiments, Cy3-conjugated human insulin (Phoenix Pharmaceuticals, Inc., Burlingame, California) diluted to $2.5 \mathrm{nM}$ in $1 \times \mathrm{DMEM}$ was incubated for $5 \mathrm{~min}$ at $37^{\circ} \mathrm{C}$ in the presence of $5 \% \mathrm{CO}_{2}$.

\subsection{Single-Molecule Imaging}

A 532-nm DPSS laser (100 mW, Cobolt Samba, Solna, Sweden) and a 488-nm argon-ion laser (Melles Grioet, Albuquerque, New Mexico) were used to excite the Cy3 and QD605, respectively. The emission signals from the fluorophores were imaged in an objective-type total internal reflection fluorescence microscope (homebuilt with an Olympus IX-71, oil-type 60× objective, $\mathrm{NA}=1.45)$ using an electron-multiplying charge-coupled device (ImageEM C9100-13, Hamamatsu) [Fig. 2(a)]. The fluorescent signals were collected with a time resolution of $50 \mathrm{~ms}$ for $100 \mathrm{~s}$ by an imaging program (HCimage, Hamamatsu). The acquired images were analyzed by MATLAB ${ }^{\circledR}$ (MathWorks, Natick, Massachusetts) scripts to determine the positions of the emitters. ${ }^{14}$

Table 1 Sequences and dissociation constants $\left(K_{d}\right)$ of IR-A07 and IR-A29.

\begin{tabular}{|c|c|c|c|c|}
\hline Id & Sequence & Size & dT modification & $K_{d}(\mathrm{nM})$ \\
\hline A07 & 5'-GCCTGBBGABBABBAACGAGABGAGCCCCBCCCBGACAACCBCACCAGCC-3' & 49 mer & $B=$ Benzyl dT & $11.6 \pm 0.5$ \\
\hline A29 & 5'-GCCTGNNAGGCAGGGNGANGCCNGCCGGNNNCGGCCAANAGCGNNCAGCC-3' & 50 mer & $\mathrm{N}=$ Napthyl dT & $33.4 \pm 0.6$ \\
\hline
\end{tabular}



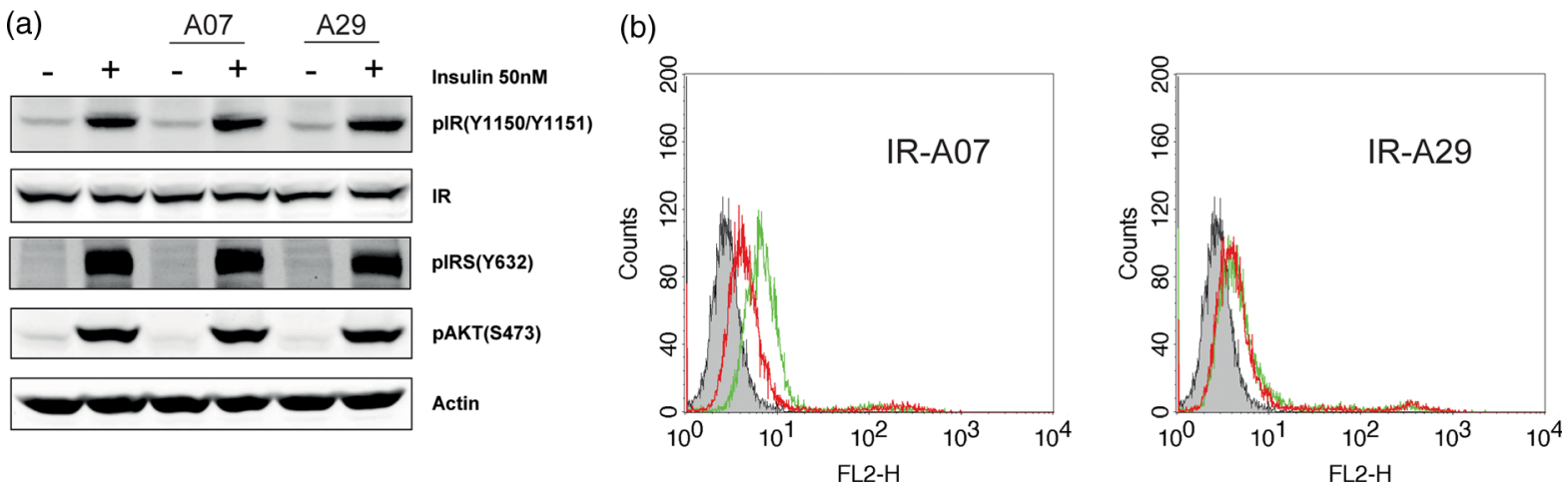

Fig. 1 Insulin receptor (IR)-specific binding aptamers are nonfunctional binders. Two IR-specific binding aptamers, namely IR-A07 and IR-A29, were selected through a SELEX process. (a) The western blot results show that IR-A07 and IR-A29 can neither activate nor inhibit insulin signaling in cells. The tests were performed by applying $50 \mathrm{nM}$ insulin and $1 \mu \mathrm{M}$ aptamers in HIR cells for $15 \mathrm{~min}$. (b) The flow cytometry results of the interruption test of IR-A07 and IR-A29 binding to IRs by insulin ligand. Black: cell only, Green: Cy3-conjugated aptamer (200 nM) only, Red: Cy3-conjugated aptamer $(200 \mathrm{nM})+$ insulin $(1 \mu \mathrm{M})$.

(a)

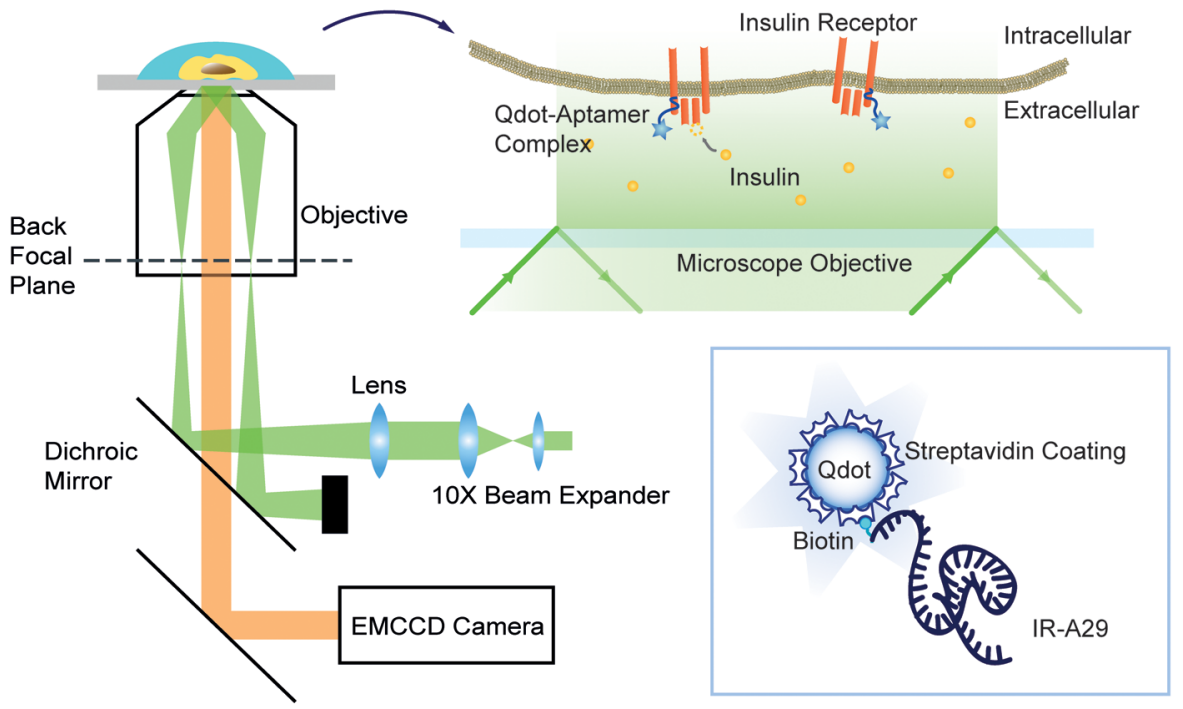

(b)

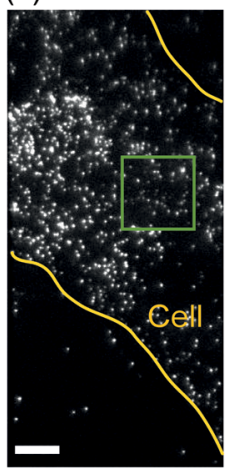

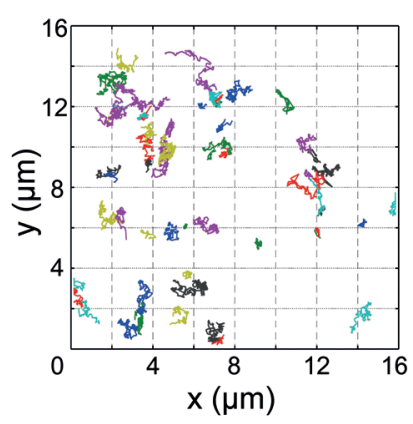

(c)

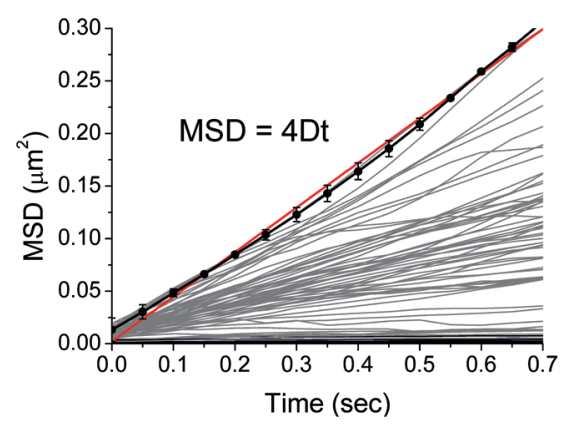

Fig. 2 Dynamics of single IRs in living cell were visualized by fluorophore-aptamer labeling and total internal fluorescence resonance (TIRF) microscopy. (a) An objective-type TIRF microscope used for imaging enables us to observe diffusing IRs on the basal cell membrane with minimal background noise. The experiment with QD aptamer labeling after the addition of ligands is shown in this schematic representation. Biotin-A29 aptamer was conjugated with streptavidin-coated QD605 for fluorescent microscopy (TIFRM) by incubating the sample with streptavidin-coated QDs in BSA-containing DMEM solution after incubation with $28 \mathrm{nM}$ biotin-A29 and BSA to increase the binding specificity. (b) By analyzing the acquired images of QD aptamer binding to HIR cells, the trajectories of molecules in a selected square region of $100 \times 100$ pixels were constructed. Specific binding events of QDs to IRs are shown in this image, with rare nonspecific binding events on the glass surface, which significantly aid in distinguishing the cells. Scale bar: $10 \mu \mathrm{m}$. (c) Using the plot of mean-square displacement (MSD) versus time interval for the trajectories, the diffusion coefficients corresponding to the slope of the plot were calculated from the linear fits. Most of the trajectories are linear, indicating that free diffusion is a major component of the IR dynamics in the plasma membranes of living cells. 


\section{Results and Discussion}

\subsection{Modified DNA Aptamers as a Probe for Targeting IRs}

We developed two modified single-stranded DNA (ssDNA) aptamers targeting IRs, IR-A07 and IR-A29, by SELEX. The IR-A07 aptamer, consisting of 49 nucleotides, was modified with benzyl thymines (dTs) and IR-A29, with 50 nucleotides, contains modified naphthyl dTs. We measured the bulk dissociation constants $\left(K_{d}\right)$ of the two aptamers for IRs using a direct binding assay (Table 1; Sec. 2). The results indicate that these aptamers with nanomolar $K_{d}$ values are good probes for detecting the target molecules. A western blot was used to determine whether the aptamers affect insulin-induced signaling in HIR cells. Figure 1(a) shows that the insulin-induced phosphorylation of IR (pY1150pY1151), IRS-1 (Y632), and AKT (S473) is neither activated nor interrupted by the two aptamers. The IR-A07 and IR-A29 aptamers do not promote insulin signaling upon binding to the receptors, which indicates that these aptamers are appropriate as neutral probes without stimulating the downstream signaling. As shown in Fig. 1(b), the flow cytometry displays that IR-A07 aptamers bound to IRs are slightly disrupted by insulin ligand binding (left), but IRbound IR-A29 aptamers do not display any interruption due to insulin binding (right). Taken together, we found that two aptamers can be used as probes for visualizing IRs with a high affinity, but IR-A29 is more suitable to study the insulindependent dynamics of IR.

\subsection{Diffusive Motion of Individual IRs in Living Cells}

To visualize IRs in the plasma membranes of living cells, a QD was conjugated to the selected aptamer, IR-A29 [Fig. 2(a)]. Aptamer-bound IRs in HIR cells imaged using an objectivetype TIRF microscope were tracked to obtain individual trajectories. Figure 2(b) shows a representative image obtained from a HIR cell in the presence of QD-labeled aptamer $(28 \mathrm{nM})$ while free aptamers in solution were stringently washed out by pipetting. The QDs allowed us to monitor individual IRs for a long time (more than several minutes) and to accurately determine the position of IRs owing to the bright emission signals. The time trajectories of the QD aptamers bound to IRs, which were determined by the two-dimensional Gaussian-fitted center of the intensity profiles, ${ }^{15}$ indicate the diffusive motion of the IRs [Fig. 2(b), rectangular region]. The mean-square displacement (MSD) of the IR trajectories was computed [Fig. 2(c)], and the resulting MSD is linearly dependent on the time interval. Thus, the diffusion coefficient $(D)$ was determined from the slope of the MSD versus time interval $(t)$ for IRs that moved in the plasma membrane, using the relation of $\mathrm{MSD}=4 D t$ [Fig. 2(c), red line].

The resulting diffusion coefficient $\left(D_{\mathrm{w} / \mathrm{oINS}}\right)$ of IR in HIR cell membrane was measured as $0.039 \pm 0.034 \mu \mathrm{m}^{2} / \mathrm{s}$ ( $n=212$; mean \pm s.d.). To investigate whether insulin ligands bound to IRs influence the diffusive motion of the receptor, we examined the diffusion mechanics of IRs in the absence and presence of $1 \mu \mathrm{M}$ insulin ligand. The diffusion coefficient of IR in the presence of $1 \mu \mathrm{M}$ insulin $\left(D_{1 \mu \mathrm{M} \text { insulin }}=\right.$ $\left.0.043 \pm 0.036 \mu \mathrm{m}^{2} / \mathrm{s} ; n=155\right)$ was nearly identical to that in the absence of insulin [Fig. 3(a)]. This insulin concentration was intended to be much higher than the physiological concentration of human insulin $(0.057 \sim 0.079 \mathrm{nM}$ between meals and
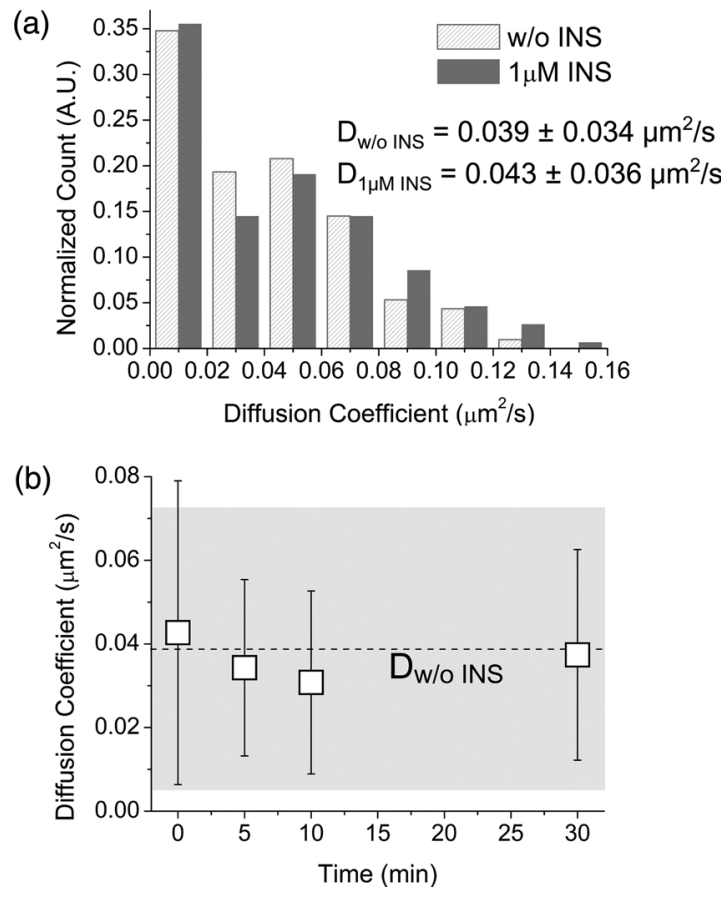

Fig. 3 The diffusion coefficients of IRs obtained from the experiment with QD aptamers were analyzed. (a) The distributions of diffusion coefficients without insulin $(n=212)$ and immediately after the injection of $1 \mu \mathrm{M}$ insulin ( 0 min after injection; $n=155)$ are shown for comparison. The distributions were normalized by dividing each column by the total number of measured diffusion coefficients. Neither the average values nor the distributions of the diffusion coefficients showed a significant change in the absence or the presence of insulin ligands. (b) Diffusion coefficients of IRs with respect to incubation time after the injection of $1 \mu \mathrm{M}$ insulin (0, 5, 10 and 30 min after injection); dotted line: diffusion coefficient without insulin ( $\left.D_{\mathrm{w} / \mathrm{oINS}}\right)$, gray area: standard deviation of $D_{w / o}$ Ins. The errors indicate the standard deviation.

up to $0.43 \mathrm{nM}$ after meals ${ }^{16}$ ) to ensure ligand binding to the IRs. To confirm insulin binding to the receptors, the diffusion coefficients were investigated for various insulin incubation times $(0,5,10$, and $30 \mathrm{~min}$ after the injection of $1 \mu \mathrm{M}$ insulin). There was no significant change in the diffusion coefficients of IR with respect to incubation times [Fig. 3(b)], which indicates that the diffusive motion of IRs may not be sensitive to insulin binding.

It has been previously reported that insulin signaling to IRs results in phosphorylation and sequential binding of various cofactors, such as SHC, Grb2, SOS, and Ras proteins. ${ }^{17}$ The Stokes-Einstein relation $\left(D=k_{\mathrm{B}} T / 6 \pi \eta r, r\right.$ : radius of the spherical object, $\eta$ : viscosity of the medium, $k_{\mathrm{B}} T$ : thermal energy) predicts that an increased complex size in the cytoplasmic region should correspond to a decreased diffusion coefficient of the IR. However, our result does not agree with this prediction. We interpret it as a much higher viscosity of the cell membrane in comparison to the intracellular viscosity may explain the invariant motion. For instance, the viscosity of the plasma membrane of living cells ranges from 30 to $200 \mathrm{cp}^{18,19}$ ( $1 \mathrm{cp}$ for water) and the intracellular viscosity is 1 to $2 \mathrm{cp}^{20}$

\subsection{Diffusive Dynamics of IRs on a Cholesterol- Depleted Membrane}

We also measured the diffusion coefficients of IRs in the various cell plasma membranes (Table 2). The IRs that were probed with 
Table 2 Diffusion coefficients of IRs in various cell membranes in the absence and presence of insulin ligands (unit: $\mu \mathrm{m}^{2} / \mathrm{s}$ ).

\begin{tabular}{lcc}
\hline & \multicolumn{2}{c}{ Insulin Ligand } \\
\cline { 2 - 3 } Cells & - & + \\
\hline HIR & $0.039 \pm 0.034(n=212)$ & $0.043 \pm 0.036(n=155)$ \\
HEK293T & $0.045 \pm 0.047(n=253)$ & $0.039 \pm 0.047(n=206)$ \\
HepG2 & $0.044 \pm 0.029(n=144)$ & $0.036 \pm 0.029(n=105)$ \\
\hline
\end{tabular}

QD-A29 aptamers were imaged at three cell lines (HIR, HEK293T, HepG2) in the absence and presence of $1 \mu \mathrm{M}$ insulin ligand. The presence of insulin ligands results in about $15 \%$ decrease of diffusion coefficients of IRs at HEK293T and HepG2 cell lines (normal cell lines) while insulin increases $10 \%$ of IR diffusion coefficient at HIR cell (an IR-overexpressing cell line) (Table 2). However, the difference of the diffusion coefficients is trivial, considering the heterogeneous distribution of IRs [Fig. 3(a)]. The diffusion coefficients also do not vary significantly with cell types. These results suggest that the IR diffusion in dominant structures may result in the independency of insulin ligand and cells.

To further explore the structured-membrane-dependent diffusion of IRs, we examined the movements of QD aptamer-bound IRs in HIR cells using methyl- $\beta$-cyclodextrin $(\mathrm{M} \beta \mathrm{CD})$ treatment, which results in the depletion of cholesterol molecules from the plasma membrane. ${ }^{10,21}$ For this observation, 5 and $10 \mathrm{mM} \mathrm{M} \beta \mathrm{CD}$ (Sigma-Aldrich) in $1 \times$ DMEM were incubated in the sample chamber for $50 \mathrm{~min}$ at $37^{\circ} \mathrm{C}$ in the presence of $5 \%$ $\mathrm{CO}_{2}$ before the aptamer binding step. Cholesterol molecules are abundant in cell membranes and modulate the fluidity of the membranes, which play a critical role in cell signaling and intracellular transport. Figure 4 shows that the cholesterol depletion of the plasma membrane resulted in a decrease in the diffusion coefficients of the IRs with increasing $\mathrm{M} \beta \mathrm{CD}$ levels; $D=0.039 \pm 0.034 \mu \mathrm{m}^{2} / \mathrm{s}$ in the absence of $\mathrm{M} \beta \mathrm{CD}$, $D=0.022 \pm 0.021 \mu \mathrm{m}^{2} / \mathrm{s}$ at $5 \mathrm{mM} \mathrm{M} \beta \mathrm{CD}$, and $D=0.013 \pm$ $0.011 \mu \mathrm{m}^{2} / \mathrm{s}$ at $10 \mathrm{mM} \mathrm{M} \beta \mathrm{CD}$. This observation indicates that the elimination of cholesterol from the plasma membrane decelerates the diffusive motion of IRs, which is similar to

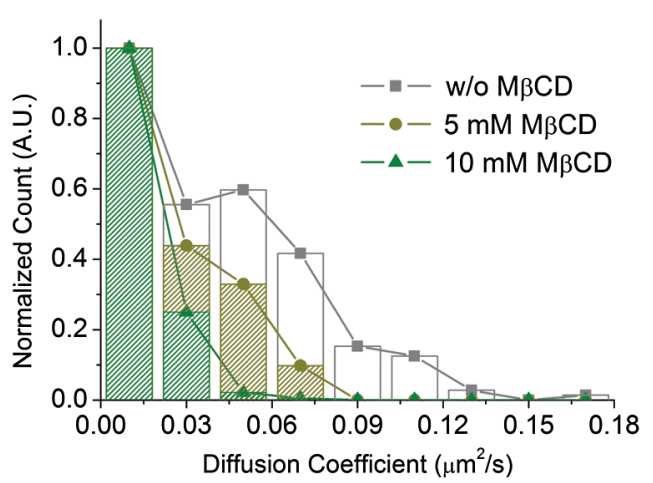

Fig. 4 Cholesterol depletion of the plasma membrane of HIR cells was induced by $\mathrm{M} \beta \mathrm{CD}$. The distribution of diffusion coefficients for various concentrations of $\mathrm{M} \beta \mathrm{CD}(n=208$ for no $\mathrm{M} \beta \mathrm{CD}, n=153$ for $5 \mathrm{mM}$ $\mathrm{M} \beta \mathrm{CD}$, and $n=235$ for $10 \mathrm{mM} M \beta C D)$. To facilitate the comparison, the distributions were normalized by setting the first column to unity.
Ras protein diffusion in cholesterol depletion with $\mathrm{M} \beta \mathrm{CD} .^{22}$ Cholesterol depletion study in dioleoylphosphatidylcholine and sphingomyelin indicates that cholesterol depletion in saturated sphingolipids decreases the fluidity of the membrane while it increases the membrane fluidity in unsaturated phospholipids. $^{23}$ Therefore, our results suggest that IRs are located at the region enriched in spingolipids and cholesterol molecules, which supports the mechanism of insulin signaling via spingolipids-cholesterol microdomains. ${ }^{24,25}$

\section{Conclusion}

We successfully selected nonfunctional ssDNA aptamers with nanomolar binding affinity to IRs through the SELEX process. The QD-labeled DNA aptamers were used to visualize individual IRs in the plasma membranes of various kinds of biologically functional cells (HEK293T, HepG2) as well as IR overexpressed cells (HIR), which allowed us to study the real-time dynamics of individual IRs in the plasma membranes of living cells. This developed single-molecule imaging method can be used for various approaches to investigate the heterogeneous nature of living cells through simple modifications of the aptamer.

\section{Acknowledgments}

We thank Dong-wook Kim for performing the initial study of living cell imaging. This work was supported by the National Research Foundation of Korea (NRF) grant funded by the Korea government (MEST) (No. 2011-0013901 for J.B.L and No. 2013R1A2A1A03010110 for S.H.R).

\section{References}

1. S. Wieser and G. J. Schütz, "Tracking single molecules in the live cell plasma membrane-Do's and Don't's," Methods 46, 131-140 (2008).

2. J. T. Groves, R. Parthasarathy, and M. B. Forstner, "Fluorescence imaging of membrane dynamics," Annu. Rev. Biomed. Eng. 10, 311-338 (2008).

3. Y. Sako, S. Minoghchi, and T. Yanagida, "Single-molecule imaging of EGFR signalling on the surface of living cells," Nat. Cell Biol. 2, 168172 (2000).

4. X. Michalet et al., "Quantum dots for live cells, in vivo imaging, and diagnostics," Science 307, 538-544 (2005).

5. N. C. Shaner, P. A. Steinbach, and R. Y. Tsien, "A guide to choosing fluorescent proteins," Nat. Methods 2, 905-909 (2005).

6. A. D. Ellington and J. W. Szostak, "In vitro selection of RNA molecules that bind specific ligands," Nature 346, 818-822 (1990).

7. C. Tuerk and L. Gold, "Systematic evolution of ligands by exponential enrichment: RNA ligands to bacteriophage T4 DNA polymerase," Science 249, 505-510 (1990).

8. W. Li et al., "Real-time imaging of protein internalization using aptamer conjugates," Anal. Chem. 80, 5002-5008 (2008).

9. A. R. Saltiel and C. R. Kahn, "Insulin signalling and the regulation of glucose and lipid metabolism," Nature 414, 799-806 (2001).

10. S. Parpal et al., "Cholesterol depletion disrupts caveolae and insulin receptor signaling for metabolic control via insulin receptor substrate1, but not for mitogen-activated protein kinase control," J. Biol. Chem. 276, 9670-9678 (2001).

11. P. A. Tulloch et al., "Single-molecule imaging of human insulin receptor ectodomain and its Fab complexes," J. Struct. Biol. 125, 11-18 (1999).

12. A. Slade et al., "Single molecule imaging of supported planar lipid bilayer-reconstituted human insulin receptors by in situ scanning probe microscopy," J. Struct. Biol. 137, 283-291 (2002).

13. Y. J. Lee et al., "Periostin-binding DNA aptamer inhibits breast cancer growth and metastasis," Mol. Ther. 21, 1004-1013 (2013).

14. W.-K. Cho et al., "ATP alters the diffusion mechanics of MutS on mismatched DNA," Structure 20, 1264 (2012). 
15. R. E. Thompson, D. R. Larson, and W. W. Webb, "Precise nanometer localization analysis for individual fluorescent probes," Biophys. J. 82, 2775-2783 (2002).

16. H. Iwase et al., "The ratio of insulin to C-peptide can be used to make a forensic diagnosis of exogenous insulin overdosage," Forensic Sci. Int. 115, 123-127 (2001).

17. J. A. Olivares-Reyes, A. Arellano-Plancarte, and J. R. CastilloHernandez, "Angiotensin II and the development of insulin resistance: implications for diabetes," Mol. Cell. Endocrin. 302, 128-139 (2009).

18. W. R. Dunham et al., "EPR measurements showing that plasma membrane viscosity can vary from 30 to $100 \mathrm{cP}$ in human epidermal cell strains," Spectrochim Acta A 52, 1357-1368 (1996).

19. J. Kapitulnik et al., "Fetal and adult human-liver differ markedly in the fluidity and lipid-composition of their microsomal-membranes," Hepatology 7, 55-60 (1987).

20. S.-P. Williams, P. M. Haggie, and K. M. Brindle, "19F NMR measurements of the rotational mobility of proteins in vivo," Biophys. J. 72, 490-498 (1997).

21. J. Shawn Goodwin et al., "Ras diffusion is sensitive to plasma membrane viscosity," Biophys. J. 89, 1398-1410 (2005).

22. J. S. Goodwin et al., "Ras diffusion is sensitive to plasma membrane viscosity," Biophys. J. 89, 1398-1410 (2005).

23. N. Kahya et al., "Probing lipid mobility of raft-exhibiting model membranes by fluorescence correlation spectroscopy," J. Biol. Chem. 278, 28109-28115 (2003).
24. P. E. Bickel, "Lipid rafts and insulin signaling," Am. J. Physiol. Endocrinol. Metab. 282, E1-E10 (2002).

25. E. Ikonen and S. Vainio, "Lipid microdomains and insulin resistance: is there a connection?," Sci. STKE 268, pe3 (2005).

Minhyeok Chang is a PhD candidate in physics at Pohang University of Science \& Technology (POSTECH).

Mijin Kwon is a research associate at Ulsan National Institute of Science \& Technology after her postdoctoral training at POSTECH.

Sooran Kim is a PhD student in physics at POSTECH.

Na-Oh Yuun is a PhD candidate in the School of Interdisciplinary Bioscience \& Bioengineering at POSTECH.

Daehyung Kim is a PhD student in physics at POSTECH.

Sung Ho Ryu is a professor of life sciences and the principal investigator of Signal Transduction Lab (www.postech.ac.kr/life/st) at POSTECH.

Jong-Bong Lee is an associate professor of physics and the principal investigator of the Single-molecule Biophysics Lab (jblab.postech.ac.kr) at POSTECH. 